\title{
Robotic Techniques in Esophagogastric Cancer Surgery: An Assessment of Short- and Long-Term Clinical Outcomes
}

\author{
Sivesh K. Kamarajah, BMedSci, MBChB, MRCS $^{1,2}$ (1) , Ewen A. Griffiths, MD, FRCS ${ }^{1,2}$, \\ Alexander W. Phillips, MD, FRCSEd ${ }^{3,4}$, Jelle Ruurda, MD $^{5}$, Richard van Hillegersberg, MD $^{5}$, \\ Wayne L. Hofstetter, MD ${ }^{6}$, and Sheraz R. Markar, MRCS, MSc, MA, PhD ${ }^{7,8}$
}

${ }^{1}$ Department of Upper Gastrointestinal Surgery, Queen Elizabeth Hospital Birmingham, University Hospitals Birmingham NHS Trust, Birmingham, UK; ${ }^{2}$ Institute of Cancer and Genomic Sciences, College of Medical and Dental Sciences, University of Birmingham, Birmingham, UK; ${ }^{3}$ Northern Oesophagogastric Unit, Royal Victoria Infirmary, Newcastle University Trust Hospitals, Newcastle Upon Tyne, UK; ${ }^{4}$ School of Medical Education, Newcastle University, Newcastle Upon Tyne, Tyne and Wear, UK; ${ }^{5}$ University Medical Center Utrecht, University Utrecht, Utrecht, The Netherlands; ${ }^{6}$ Thoracic and Cardiovascular Surgery, The University of Texas MD Anderson Cancer Center, Houston, TX; ${ }^{7}$ Department of Surgery and Cancer, Imperial College London, London, UK; ${ }^{8}$ Department of Molecular Medicine and Surgery, Karolinska Institutet, Stockholm, Sweden

\begin{abstract}
Background. Robotic esophagogastric cancer surgery is gaining widespread adoption. This population-based cohort study aimed to compare rates of textbook outcomes (TOs) and survival from robotic minimally invasive techniques for esophagogastric cancer.

Methods. Data from the United States National Cancer Database (NCDB) (2010-2017) were used to identify patients with non-metastatic esophageal or gastric cancer receiving open surgery (to the esophagus, $n=11,442$; stomach, $n=22,183$ ), laparoscopic surgery (to the esophagus [LAMIE], $n=4827$; stomach [LAMIG], $n=6359$ ), or robotic surgery (to the esophagus [RAMIE], $n=1657$; stomach [RAMIG], $n=1718$ ). The study defined TOs as 15 or more lymph nodes examined, margin-negative resections, hospital stay less than 21 days, no 30-day readmissions, and no 90-day mortalities. Multivariable logistic regression and Cox analyses were used to account for treatment selection bias.
\end{abstract}

(C) The Author(s) 2021

First Received: 17 June 2021

Accepted: 28 October 2021;

Published Online: 10 December 2021

S. R. Markar, MRCS, MSc, MA, PhD

e-mail: s.markar@imperial.ac.uk
Results. Patients receiving robotic surgery were more commonly treated in high-volume academic centers with advanced clinical $\mathrm{T}$ and $\mathrm{N}$ stage disease. From 2010 to 2017, TO rates increased for esophageal and gastric cancer treated via all surgical techniques. Compared with open surgery, significantly higher TO rates were associated with RAMIE (odds ratio [OR], 1.41; 95\% confidence interval [CI], 1.27-1.58) and RAMIG (OR 1.30; 95\% CI 1.17-1.45). For esophagectomy, long-term survival was associated with both TO (hazard ratio [HR 0.64, 95\% CI 0.60-0.67) and RAMIE (HR 0.92; 95\% CI 0.84-1.00). For gastrectomy, long-term survival was associated with TO (HR 0.58; 95\% CI 0.56-0.60) and both LAMIG (HR 0.89; 95\% CI 0.85-0.94) and RAMIG (HR 0.88; 95\% CI 0.81-0.96). Subset analysis in high-volume centers confirmed similar findings.

Conclusion. Despite potentially adverse learning curve effects and more advanced tumor stages captured during the study period, both RAMIE and RAMIG performed in mostly high-volume centers were associated with improved TO and long-term survival. Therefore, consideration for wider adoption but a well-designed phase 3 randomized controlled trial (RCT) is required for a full evaluation of the benefits conferred by robotic techniques for esophageal and gastric cancers. 
Esophagectomy and gastrectomy remain the mainstay of curative therapy for esophageal and gastric cancers, but surgical approach techniques vary. A growing evidence base for minimally invasive techniques appears to suggest either improved or similar morbidity without a compromise of oncologic quality. ${ }^{1-3}$ However, most of the studies preclude analysis comparing robotic esophagectomy or gastrectomy, which in more recent years have been gaining increasing adoption internationally.

Robotic techniques have specific advantages including a three-dimensional view and increased degrees of freedom at the wrist, which may lend to better technical surgical performance and thus better clinical outcomes for esophagectomy and gastrectomy. To date, only one singlecenter European randomized controlled trial $(\mathrm{RCT})^{4}$ has shown improvements in postoperative complications, pain, short-term quality of life, and functional recovery with robotic versus open esophagectomy. Furthermore, a recent publication from the Upper Gastrointestinal International Robotic-Assisted Association (UGIRA) highlighted promising results from this technique when undertaken in high-volume specialized centers with adequate training. ${ }^{5}$

The data concerning robotic gastrectomy is based largely on observational cohort studies ${ }^{6,7}$ and originates from the Far East, with a different patient population and standard of lymphadenectomy from what is commonly observed in Western centers.

The quality assessment of surgical care has moved toward the use of textbook outcomes (TOs), a composite measure first developed in 2010 by colorectal surgeons in Netherlands as a more reliable global metric for assessing health care quality than individual outcome parameters. ${ }^{1,8,9}$ Although data on TOs have been reported across a variety of complex surgeries such as hepatectomy, pancreatectomy, and abdominal aneurysm repair. ${ }^{1,2,8-10}$ international data for esophagogastric surgery by surgical approach is more limited. ${ }^{11-13}$

The current population-based cohort study aimed to assess the short- and long-term clinical outcomes associated with the use of robotic minimally invasive techniques for the treatment of esophageal and gastric cancers in the United States.

\section{METHODS}

\section{Data Source}

The National Cancer Database (NCDB) is a joint project of the Commission on Cancer (CoC) of the American College of Surgeons and the American Cancer Society. ${ }^{14,15}$ The NCDB gathers information from approximately 1500 CoC-accredited hospitals and includes more than $70 \%$ of all newly diagnosed malignancies in the United States of America (USA). It contains specific details about patient demographics (age, sex, race, insurance status), facility type and location, tumor characteristics (size, grade, stage, histology), treatment course (type of surgery, receipt of chemotherapy, and radiation therapy), and outcomes (resection margins, lymph node status, hospital length of stay, short- and long-term mortality).

\section{Study Population}

Inclusion Criteria The current study enrolled any patients with a diagnosis of non-metastatic esophageal (i.e., adenocarcinoma, squamous cell carcinoma) or gastric adenocarcinoma (Supplementary Table S1)) according to the International Classification of Disease for Oncology, third edition (ICD-O-3) who received esophagectomy or gastrectomy between 2010 and 2016 in the de-identified NCDB.

Exclusion Criteria The exclusion criteria ruled out other histology subtypes such as mucinous tumors, neuroendocrine tumors, and other histologies; patients who underwent endoscopic resection; other concurrent cancer diagnoses; and patients with metastatic esophageal or gastric cancer.

\section{Study Definitions}

The following patient-level characteristics as provided by NCDB were analyzed: age $(18-35,36-50,51-65,66-$ $80, \geq 81$ years), race (white, other), Charlson/Deyo comorbidity score, ${ }^{16}$ year of diagnosis, insurance status (Medicare, Medicaid, private insurance, uninsured), zip code-level education status $(<7 \%, 7-12.9 \%, 13-20.9 \%, \geq$ $21 \%)$, zip code-level median household income $(<$ $\$ 48,000, \$ 48,000-62,999$, $\geq \$ 63,000$ ), and urban versus rural area of residence. The zip code level education status represents the proportion of adults in the patient's zip code who did not graduate from high school categorized as equally proportioned quartiles among all U.S. zip codes.

The following hospital-level characteristics also were analyzed: center volume (quintiles 1-5), facility type (academic, community, other), facility location (Midwest, Northeast, South, West), and hospital distance from the patient's residence $(<12.5,12.5-49.9, \geq 50.0$ miles $)$. Center volume was defined according to the annual surgical volume at each individual hospital derived from the unique center identification number. These then were split into five equal groups for center volume.

Finally, the study analyzed the following clinicopathologic characteristics: clinical $\mathrm{T}$ status (T1, T2, T3, T4, Tx) and $\mathrm{N}$ status (N0, N1, N2, N3, Nx), receipt of neoadjuvant 
therapy, tumor grade/differentiation (well/moderate, poor/ anaplastic), margin status (positive, negative), and lymphovascular invasion (absent, present, unknown). Neoadjuvant therapy was defined as none, neoadjuvant chemotherapy, or neoadjuvant chemoradiotherapy. In both the group receiving neoadjuvant chemotherapy and the group receiving chemoradiotherapy, only patients receiving multi-agent chemotherapy were considered.

\section{Outcome Measures}

The primary outcome was the rate of TOs, with TO defined as margin-negative resections, 15 or more lymph nodes examined, no prolonged hospital stay ( $\geq 21$ days), no 90-day postoperative mortality, and no readmission 30 days or less after discharge. A TO was achieved when all these parameters were fulfilled. The definition used for TO in this study was derived from the original study by Busweiler et al. ${ }^{17}$ The secondary outcome was long-term survival, defined as the time from surgery to the last known follow-up visit or death.

\section{Statistical Analysis}

Categorical variables were compared using the chisquare test. Non-normally distributed data were analyzed using the Mann-Whitney $U$ test. Survival was estimated using Kaplan-Meier survival curves and compared using the log-rank test. A multilevel logistic regression model was used to produce an adjusted odds ratio (OR) and a $95 \%$ confidence interval $(95 \% \mathrm{CI})$ to determine the association between surgical approach and TO. Multivariable analyses used Cox proportional hazards models.

In all models, patient-level, hospital-level, and tumorlevel characteristics were included. Importantly, year of diagnosis was included to adjust for changes in developments in patient selection, diagnostic staging, multimodality treatment, and perioperative care (e.g., prehabilitation). Subset analyses were performed in highvolume centers to assess the impact of open, laparoscopic, and robotic techniques on TO and survival for both esophagectomy and gastrectomy patients. Post hoc analyses also were performed to compare outcomes for laparoscopic and robotic techniques.

A $p$ value lower than 0.05 was considered statistically significant. Data analysis was performed using R Foundation Statistical software (R 3.2.2) with TableOne, ggplot2, Hmisc, Matchit and survival packages (R Foundation for Statistical Computing, Vienna, Austria), as previously reported. $^{18}$

\section{RESULTS}

\section{Esophageal Cancer}

Baseline Characteristics This study identified 17,947 patients with esophageal cancer treated with esophagectomy, of which $27 \%(n=4827)$ were a conventional total or hybrid minimally invasive esophagectomy (LAMIE) and 9\% $(n=1,657)$ were a robot-assisted minimally invasive esophagectomy (RAMIE). The patients receiving RAMIE were more commonly treated in high-volume centers $(27 \%$ vs $24 \%$ vs $23 \% ; p<0.001)$ and academic centers (67\% vs $65 \%$ vs $59 \% ; p<0.001)$ compared with LAMIE and open surgery. Furthermore, the patients receiving RAMIE were more likely older patients with advanced $\mathrm{T}$ and $\mathrm{N}$ stage than those treated with conventional LAMIE or open surgery. Baseline demographics are presented in Table 1.

Textbook Outcome (TO) The individual rates of TO parameters were $94 \%$ for margin-negative resections, $46 \%$ for 15 or more lymph nodes examined, $86 \%$ for a hospital stay of 21 days or less, $92 \%$ for no 30-day readmission, and $93 \%$ for no 90 -day postoperative mortality. The individual rate of TO parameters was significantly higher with RAMIE than with LAMIE or open esophagectomy for margin-negative resections (96\% vs $95 \%$ vs $93 \% ; p<$ $0.001), 15$ or more lymph nodes examined (55\% vs $53 \%$ vs $42 \% ; p<0.001)$, and hospital stay of 21 days or less $(88 \%$ vs $88 \%$ vs $85 \%$; $p<0.001)$, but did not differ for 30 -day readmissions (Table 2 ).

The overall prevalence of TOs observed was $36 \%(n=$ 6,528 ) and significantly higher for the patients receiving RAMIE than for those receiving LAMIE and or open surgery (44\% vs $43 \%$ vs $32 \%$; $p<0.001$ ) (Tables 2,3 ). In the adjusted analysis, the patients receiving either LAMIE (OR, 1.35, 95\% CI 1.26-1.46; $p<0.001$ ) or RAMIE (OR, $1.41 ; 95 \%$ CI $1.27-1.58 ; p<0.001)$ had a greater proportion of TOs than those treated with open esophagectomy (Supplementary Table S2).

Association Between TO and Survival The patients achieving TO had a significantly longer survival than those without TO (median, 70.5 vs 38.2 months; $p<0.001$ ) (Fig. 1A). The patients receiving RAMIE or LAMIE had a significantly longer survival than those treated with open esophagectomy (median, 56.7 vs 54.4 vs 45.0 months; $p<$ 0.001; Fig. 1B). In the adjusted analyses, significantly improved overall survival was associated with TO (hazard ratio [HR], 0.64; 95\% CI 0.60-0.67; $p<0.001)$ and RAMIE (HR 0.92; 95\% CI 0.84-1.00; $p=0.049$ ) (Tables 3 and Supplementary S3). However, long-term survival did 
TABLE 1 Baseline clinicopathologic characteristics of patients undergoing esophagectomy for esophageal cancer by surgical approach

\begin{tabular}{|c|c|c|c|c|}
\hline & $\begin{array}{l}\text { Open } \\
(n=11,463) \\
n(\%)\end{array}$ & $\begin{array}{l}\text { Laparoscopic } \\
(n=4827) \\
n(\%)\end{array}$ & $\begin{array}{l}\text { Robotic } \\
(n=1657) \\
n(\%)\end{array}$ & $p$ value \\
\hline \multicolumn{5}{|l|}{ Center volume } \\
\hline 1 (Lowest) & $1422(12.4)$ & $400(8.3)$ & $128(7.7)$ & \multirow[t]{5}{*}{$<0.001$} \\
\hline 2 & $2062(18.0)$ & 758 (15.7) & 309 (18.6) & \\
\hline 3 & $2632(23.0)$ & 1056 (21.9) & $376(22.7)$ & \\
\hline 4 & $2749(24.0)$ & 1433 (29.7) & 396 (23.9) & \\
\hline 5 (Highest) & 2584 (22.6) & $1177(24.4)$ & $450(27.1)$ & \\
\hline \multicolumn{5}{|l|}{ Facility type } \\
\hline Community & $3225(28.1)$ & $1076(22.3)$ & $302(18.2)$ & \multirow[t]{3}{*}{$<0.001$} \\
\hline Integrated & 1474 (12.9) & $612(12.7)$ & $251(15.1)$ & \\
\hline Academic & $6758(59.0)$ & $3143(65.1)$ & $1106(66.7)$ & \\
\hline \multicolumn{5}{|l|}{ Facility location } \\
\hline Northeast & 2277 (19.9) & 1477 (30.6) & 369 (22.2) & \multirow[t]{4}{*}{$<0.001$} \\
\hline Midwest & 3704 (32.3) & $1116(23.1)$ & $404(24.4)$ & \\
\hline South & 3943 (34.4) & $1352(28.0)$ & $669(40.3)$ & \\
\hline West & 1533 (13.4) & 886 (18.3) & $217(13.1)$ & \\
\hline \multicolumn{5}{|c|}{ Hospital distance (from patient's residence) miles } \\
\hline$<12.5$ & 4789 (41.8) & $1995(41.3)$ & $704(42.4)$ & \multirow[t]{3}{*}{0.7} \\
\hline $12.5-49.9$ & $3729(32.5)$ & $1615(33.4)$ & $551(33.2)$ & \\
\hline$\geq 50$ miles & $2939(25.7)$ & $1221(25.3)$ & $404(24.4)$ & \\
\hline \multicolumn{5}{|l|}{ Year of diagnosis } \\
\hline 2010-2011 & $3291(28.7)$ & $934(19.3)$ & $162(9.8)$ & \multirow[t]{4}{*}{$<0.001$} \\
\hline 2012-2013 & $3032(26.5)$ & $1100(22.8)$ & $354(21.3)$ & \\
\hline 2014-2015 & $1414(12.3)$ & $605(12.5)$ & $260(15.7)$ & \\
\hline 2016-2017 & $3720(32.5)$ & $2192(45.4)$ & $883(53.2)$ & \\
\hline \multicolumn{5}{|c|}{ Age at diagnosis (years) } \\
\hline $18-35$ & $76(0.7)$ & $27(0.6)$ & $10(0.6)$ & \multirow[t]{5}{*}{0.036} \\
\hline $36-50$ & $967(8.5)$ & $378(7.8)$ & $124(7.5)$ & \\
\hline $51-65$ & 5555 (48.6) & $2279(47.3)$ & $746(45.0)$ & \\
\hline $66-80$ & $4585(40.1)$ & $2024(42.0)$ & $736(44.4)$ & \\
\hline $80+$ & $256(2.2)$ & $114(2.4)$ & $42(2.5)$ & \\
\hline \multicolumn{5}{|l|}{ Sex } \\
\hline Male & $9397(82.0)$ & $3985(82.5)$ & $1378(83.1)$ & \multirow[t]{2}{*}{0.5} \\
\hline Female & $2060(18.0)$ & $846(17.5)$ & $281(16.9)$ & \\
\hline \multicolumn{5}{|l|}{ Race } \\
\hline White & $10501(91.7)$ & $4485(92.8)$ & 1517 (91.4) & \multirow[t]{2}{*}{0.029} \\
\hline Other & $956(8.3)$ & $346(7.2)$ & $142(8.6)$ & \\
\hline \multicolumn{5}{|l|}{ CDCC score } \\
\hline 0 & $7950(69.4)$ & $3311(68.5)$ & $1167(70.3)$ & \multirow[t]{4}{*}{0.3} \\
\hline 1 & $2609(22.8)$ & $1116(23.1)$ & $373(22.5)$ & \\
\hline 2 & $634(5.5)$ & $288(6.0)$ & $73(4.4)$ & \\
\hline $3+$ & $264(2.3)$ & $116(2.4)$ & $46(2.8)$ & \\
\hline \multicolumn{5}{|l|}{ Insurance status } \\
\hline Medicare & $5099(45.4)$ & $2182(46.0)$ & $818(50.2)$ & \multirow[t]{4}{*}{$<0.001$} \\
\hline Medicaid & $695(6.2)$ & $306(6.5)$ & $83(5.1)$ & \\
\hline Private & 4927 (43.9) & $2102(44.4)$ & $691(42.4)$ & \\
\hline Not insured/other & $507(4.5)$ & $149(3.1)$ & $36(2.2)$ & \\
\hline
\end{tabular}


TABLE 1 (continued)

\begin{tabular}{|c|c|c|c|c|}
\hline & $\begin{array}{l}\text { Open } \\
(n=11,463) \\
n(\%)\end{array}$ & $\begin{array}{l}\text { Laparoscopic } \\
(n=4827) \\
n(\%)\end{array}$ & $\begin{array}{l}\text { Robotic } \\
(n=1657) \\
n(\%)\end{array}$ & $p$ value \\
\hline \multicolumn{5}{|c|}{ Education level (\%) } \\
\hline$>21$ & $2584(22.6)$ & $1146(23.7)$ & $408(24.6)$ & \multirow[t]{4}{*}{0.007} \\
\hline $13-20.9$ & $2750(24.0)$ & $1033(21.4)$ & $382(23.0)$ & \\
\hline $7-12.9$ & $3612(31.5)$ & $1530(31.7)$ & $520(31.3)$ & \\
\hline$<7$ & $2511(21.9)$ & $1122(23.2)$ & $349(21.0)$ & \\
\hline \multicolumn{5}{|c|}{ Medical income (\$) } \\
\hline$\leq 47,999$ & $4154(36.3)$ & 1577 (32.6) & $550(33.2)$ & \multirow[t]{3}{*}{$<0.001$} \\
\hline $48,000-62,999$ & $2927(25.5)$ & $1201(24.9)$ & $403(24.3)$ & \\
\hline $63,000+$ & $4376(38.2)$ & $2053(42.5)$ & $706(42.6)$ & \\
\hline \multicolumn{5}{|l|}{ Residence } \\
\hline Metro & 8656 (75.6) & $3786(78.4)$ & $1362(82.1)$ & \multirow[t]{3}{*}{$<0.001$} \\
\hline Urban & $1967(17.2)$ & $737(15.3)$ & $182(11.0)$ & \\
\hline Rural & $834(7.3)$ & $308(6.4)$ & $115(6.9)$ & \\
\hline \multicolumn{5}{|c|}{ AJCC clinical T stage } \\
\hline cT1 & $1920(16.8)$ & 947 (19.6) & $244(14.7)$ & \multirow[t]{5}{*}{$<0.001$} \\
\hline $\mathrm{cT} 2$ & $2041(17.8)$ & 913 (18.9) & $304(18.3)$ & \\
\hline cT3 & $5699(49.7)$ & $2338(48.4)$ & $917(55.3)$ & \\
\hline cT4 & $253(2.2)$ & $80(1.7)$ & $29(1.7)$ & \\
\hline cTx & $1544(13.5)$ & $553(11.4)$ & $165(9.9)$ & \\
\hline \multicolumn{5}{|c|}{ AJCC clinical $N$ stage } \\
\hline cN0 & $5405(47.2)$ & $2336(48.4)$ & $798(48.1)$ & \multirow[t]{5}{*}{$<0.001$} \\
\hline $\mathrm{cN} 1$ & $4021(35.1)$ & $1678(34.7)$ & $605(36.5)$ & \\
\hline $\mathrm{cN} 2$ & $1110(9.7)$ & $494(10.2)$ & $174(10.5)$ & \\
\hline $\mathrm{cN3}$ & $208(1.8)$ & $84(1.7)$ & $23(1.4)$ & \\
\hline $\mathrm{cNx}$ & $713(6.2)$ & $239(4.9)$ & $59(3.6)$ & \\
\hline \multicolumn{5}{|l|}{ Histology } \\
\hline Adenocarcinoma & $9255(80.8)$ & $3967(82.1)$ & $1367(82.4)$ & \multirow[t]{2}{*}{0.065} \\
\hline $\mathrm{SCC}$ & $2202(19.2)$ & 864 (17.9) & $292(17.6)$ & \\
\hline \multicolumn{5}{|c|}{ Neoadjuvant therapy } \\
\hline None & $3313(28.9)$ & $1470(30.4)$ & $373(22.5)$ & \multirow[t]{3}{*}{$<0.001$} \\
\hline NCRT & $6888(60.1)$ & $2895(59.9)$ & $1116(67.3)$ & \\
\hline NAC & $1256(11.0)$ & $466(9.6)$ & $170(10.2)$ & \\
\hline \multicolumn{5}{|l|}{ Tumor grade } \\
\hline Well & $727(6.3)$ & $402(8.3)$ & $115(6.9)$ & \multirow[t]{4}{*}{$<0.001$} \\
\hline Moderate & 4519 (39.4) & $1937(40.1)$ & $647(39.0)$ & \\
\hline Poor & 4539 (39.6) & $1822(37.7)$ & $639(38.5)$ & \\
\hline Anaplastic & $1672(14.6)$ & $670(13.9)$ & $258(15.6)$ & \\
\hline \multicolumn{5}{|c|}{ AJCC pathologic T stage } \\
\hline pT0 & $2084(18.2)$ & $940(19.5)$ & $383(23.1)$ & \multirow[t]{6}{*}{$<0.001$} \\
\hline pT1 & $3101(27.1)$ & $1506(31.2)$ & 475 (28.6) & \\
\hline pT2 & 1671 (14.6) & $713(14.8)$ & $254(15.3)$ & \\
\hline pT3 & 3431 (29.9) & $1291(26.7)$ & $436(26.3)$ & \\
\hline pT4 & $147(1.3)$ & $37(0.8)$ & $12(0.7)$ & \\
\hline pTx & $1023(8.9)$ & $344(7.1)$ & $99(6.0)$ & \\
\hline \multicolumn{5}{|c|}{ AJCC pathologic $N$ stage } \\
\hline pNO & 7063 (61.6) & 3108 (64.3) & $1098(66.2)$ & $<0.001$ \\
\hline
\end{tabular}


TABLE 1 (continued)

\begin{tabular}{lccc}
\hline & $\begin{array}{l}\text { Open } \\
(n=11,463) \\
n(\%)\end{array}$ & $\begin{array}{l}\text { Laparoscopic } \\
(n=4827) \\
n(\%)\end{array}$ & $\begin{array}{l}\text { Robotic } \\
(n=1657) \\
(\%)\end{array}$ \\
\hline $\mathrm{pN3}$ & $436(3.8)$ & $144(3.0)$ & $39(2.4)$ \\
$\mathrm{pNx}$ & $870(7.6)$ & $311(6.4)$ & $67(4.0)$ \\
Lymphovascular invasion & & & $949(57.2)$ \\
Absent & $6451(56.3)$ & $2842(58.8)$ & $243(14.6)$ \\
Present & $1945(17.0)$ & $747(15.5)$ & $467(28.1)$ \\
Unknown & $3061(26.7)$ & $1242(25.7)$ & $1609(97.0)$ \\
30-Day mortality & & $4723(97.8)$ & $50(3.0)$ \\
No & $11048(96.4)$ & $108(2.2)$ & $<0.001$ \\
Yes & $409(3.6)$ & & \\
\hline
\end{tabular}

CDCC Charlson-Deyo Score, AJCC American Joint Committee on Cancer, SCC squamous cell carcinoma, NCRT neoadjuvant chemoradiotherapy, $N A C$ neoadjuvant chemotherapy

not differ significantly between RAMIE and LAMIE (HR 0.92; 95\% CI 0.84-1.00; $p=0.3$; Table 3).

Sensitivity Analysis of TO Sensitivity analyses were performed accounting for length of stay $\leq 10$ days in the TO. The patients receiving RAMIE had a significantly higher TO than those treated with LAMIE or open surgery (44\% vs $43 \%$ vs $32 \% ; p<0.001$ ), which remained consistent in the adjusted analysis (Supplementary Table S2). The patients achieving TO had a significantly longer survival than those without TO (median, $71.1 \mathrm{vs}$ 42.1 months; $p<0.001)$. Adjusted analyses showed that the patients receiving RAMIE had a significantly longer survival than those treated with open esophagectomy (HR 0.91 ; 95\% CI 0.84-1.00; $p=0.048$; Supplementary Table S4).

TO High-Volume (Quintile 5) Centers Subset analyses were performed including high-volume centers $(n=4211)$, of which $28 \%$ were LAMIE and $11 \%$ were RAMIE. The baseline demographics for open surgery, LAMIE, and RAMIE are presented in Supplementary Table S5. The individual TOs are presented in Supplementary Table S6.

The individual rate of TO parameters was significantly higher with RAMIE than with LAMIE or open esophagectomy for margin-negative resections (97\% vs $94 \%$ vs $95 \% ; p=0.044$ ) and 15 or more lymph nodes examined (70\% vs $67 \%$ vs $52 \%$; $p<0.001$ ), but did not differ for length of stay or 30-day readmissions (Supplementary Table S6). The overall prevalence of TOs observed was $47 \%(n=1974)$ and significantly higher for the patients receiving RAMIE than for those treated with LAMIE or open surgery ( $59 \%$ vs $54 \%$ vs $42 \%$; $p<0.001$; Table 3).
In the adjusted analysis, only the patients receiving RAMIE had a greater proportion of TOs (OR, 1.72; 95\% CI $1.37-2.15 ; p<0.001)$ than those treated with open esophagectomy (Supplementary Table S7). The patients achieving TO had a significantly longer survival than those without TO (median, 80.4 vs 42.1 months; $p<0.001$; Supplementary Figure S1A). The patients receiving RAMIE or LAMIE had a significantly longer survival than those treated with open esophagectomy (median, 73.1 vs 57.9 vs 51.7 months; $p<0.001$; Supplementary Figure S1B).

In the adjusted analyses, significantly better overall survival was associated with TO (HR 0.58; 95\% CI $0.52-0.64 ; p<0.001$ ) and RAMIE (HR 0.81; 95\% CI 0.68-0.96; $p=0.017$ ) (Table 3, Supplementary Table S8). However, long-term survival did not differ significantly between RAMIE and LAMIE (HR 0.99; 95\% CI 0.90-1.09; $p=0.8$ ) (Table 3).

\section{Gastric Cancer}

Baseline Characteristics The study identified 30,324 patients with gastric cancer, of which $21 \%(n=6375)$ received laparoscopic surgery $(\mathrm{LAMIG})$ and $6 \%(n=1721)$ received robot-assisted minimally invasive (RAMIG). The patients receiving RAMIG were more commonly treated in high-volume centers (32\% vs $29 \%$ vs $20 \%$; $p<0.001$ ) and academic centers (58\% vs $57 \%$ vs $45 \%$; $p<0.001$ ), had a higher annual medical income, and had higher rates of neoadjuvant chemoradiotherapy/chemotherapy than those treated with LAMIG or open gastrectomy. Baseline demographics are presented in Table 4. 
TABLE 2 Individual textbook parameters for patients undergoing esophagectomy for esophageal cancer or gastrectomy for gastric cancer by surgical approach

\begin{tabular}{|c|c|c|c|c|c|}
\hline & $\begin{array}{l}\text { Open } \\
n(\%)\end{array}$ & $\begin{array}{l}\text { Laparoscopic } \\
n(\%)\end{array}$ & $\begin{array}{l}\text { Robotic } \\
n(\%)\end{array}$ & $p$ Value & $p$ Value $^{\mathrm{a}}$ \\
\hline \multicolumn{6}{|l|}{ Esophagectomy } \\
\hline \multicolumn{6}{|c|}{ Regional nodes examined } \\
\hline$<15$ & $6637(57.9)$ & $2255(46.7)$ & $754(45.4)$ & \multirow[t]{2}{*}{$<0.001$} & \multirow[t]{2}{*}{0.4} \\
\hline$\geq 15$ & $4820(42.1)$ & $2576(53.3)$ & $905(54.6)$ & & \\
\hline \multicolumn{6}{|l|}{ Margin status } \\
\hline Negative & $10697(93.4)$ & $4565(94.5)$ & $1596(96.2)$ & \multirow[t]{2}{*}{$<0.001$} & \multirow[t]{2}{*}{0.008} \\
\hline Positive & $760(6.6)$ & $266(5.5)$ & $63(3.8)$ & & \\
\hline \multicolumn{6}{|c|}{ Length of stay (days) } \\
\hline$\leq 21$ & $9782(85.4)$ & $4261(88.2)$ & $1461(88.1)$ & \multirow[t]{2}{*}{$<0.001$} & \multirow[t]{2}{*}{0.9} \\
\hline$>21$ & $1675(14.6)$ & $570(11.8)$ & $198(11.9)$ & & \\
\hline \multicolumn{6}{|l|}{ 90-Day mortality } \\
\hline No & $10582(92.4)$ & $4570(94.6)$ & $1561(94.1)$ & \multirow[t]{2}{*}{$<0.001$} & \multirow[t]{2}{*}{0.5} \\
\hline Yes & $875(7.6)$ & $261(5.4)$ & $98(5.9)$ & & \\
\hline \multicolumn{6}{|c|}{ 30-Day readmission } \\
\hline No & $10502(91.8)$ & $4476(92.7)$ & $1532(92.5)$ & \multirow[t]{3}{*}{0.172} & \multirow[t]{3}{*}{0.7} \\
\hline Yes-unplanned & $154(1.3)$ & $51(1.1)$ & $15(0.9)$ & & \\
\hline Yes-planned & $786(6.9)$ & $300(6.2)$ & $110(6.6)$ & & \\
\hline \multicolumn{6}{|l|}{ Gastrectomy } \\
\hline \multicolumn{6}{|c|}{ Regional nodes examined } \\
\hline$<15$ & $10555(47.5)$ & $2671(41.9)$ & $646(37.5)$ & \multirow[t]{2}{*}{$<0.001$} & \multirow[t]{2}{*}{0.001} \\
\hline$\geq 15$ & $11673(52.5)$ & $3704(58.1)$ & $1075(62.5)$ & & \\
\hline \multicolumn{6}{|l|}{ Margin status } \\
\hline Negative & $19272(86.7)$ & $5839(91.6)$ & $1611(93.6)$ & \multirow[t]{2}{*}{$<0.001$} & \multirow[t]{2}{*}{0.007} \\
\hline Positive & $2956(13.3)$ & $536(8.4)$ & $110(6.4)$ & & \\
\hline \multicolumn{6}{|c|}{ Hospital stay (days) } \\
\hline$\leq 21$ & $2216(10.0)$ & $534(8.4)$ & $143(8.3)$ & \multirow[t]{2}{*}{$<0.001$} & \multirow[t]{2}{*}{1.0} \\
\hline$>21$ & $20012(90.0)$ & $5841(91.6)$ & $1578(91.7)$ & & \\
\hline \multicolumn{6}{|l|}{ 90-Day mortality } \\
\hline No & 20348 (91.5) & $6024(94.5)$ & $1643(95.5)$ & \multirow[t]{2}{*}{$<0.001$} & \multirow[t]{2}{*}{0.1} \\
\hline Yes & $1880(8.5)$ & $351(5.5)$ & $78(4.5)$ & & \\
\hline 30-Day readmissi & & & & & \\
\hline No & $20194(91.0)$ & $5894(92.7)$ & $1599(93.1)$ & $<0.001$ & 0.4 \\
\hline Yes-unplanned & $398(1.8)$ & $75(1.2)$ & $14(0.8)$ & & \\
\hline Yes-planned & $1591(7.2)$ & $390(6.1)$ & $105(6.1)$ & & \\
\hline
\end{tabular}

${ }^{a}$ Indicates post hoc analyses comparing laparoscopic and robotic surgeries
TO The individual rate of TO parameters was $88 \%$ for margin-negative resections, $54 \%$ for 15 or more lymph nodes examined, $91 \%$ for hospital stay of 21 days or longer, $92 \%$ for no 30-day readmission, and $92 \%$ for no 90-day postoperative mortality. The individual rates of TO parameters were significantly higher with RAMIG than with LAMIG or open gastrectomy for margin-negative resections (94\% vs $92 \%$ vs $87 \%$; $p<0.001), 15$ or more lymph nodes examined (63\% vs $58 \%$ vs $53 \% ; p<0.001)$, and hospital stay of 21 days or longer $(92 \%$ vs $92 \%$ vs $90 \% ; p<0.001$ ) (Table 2). The overall TO rate was $41 \%$ $(n=12,542)$ and significantly higher for the patients receiving RAMIG than for those treated with LAMIG or open gastrectomy (52\% vs $48 \%$ vs $39 \%$; $p<0.001$ ) (Table 3). The adjusted analysis showed a significantly increased rate of TO for the patients receiving LAMIG (OR, 1.19; 95\% CI 1.12-1.26; $p<0.001)$ or RAMIG (OR, $1.30 ; 95 \%$ CI $1.17-1.45, p<0.001)$ than for those treated with open gastrectomy (Supplementary Table S9).

Association Between TO and Survival The patients achieving TO had significantly better survival than those without TO (median, 79.6 vs 32.6 months; $p<0.001$; Fig. 2A). The patients receiving RAMIG or LAMIG had a 
TABLE 3 Textbook outcomes and long-term survival of patients undergoing esophagectomy for esophageal cancer or gastrectomy and gastric cancer by surgical approach and stratified analysis in high-volume centers

\begin{tabular}{|c|c|c|c|c|c|c|}
\hline & $\begin{array}{l}\text { Textbook } \\
\text { outcomes } \\
n(\%)\end{array}$ & $\begin{array}{l}\text { Median overall } \\
\text { survival } \\
\text { Months (range) }\end{array}$ & $\begin{array}{l}\text { Adjusted HR }(95 \% \\
\text { CI) }\end{array}$ & $p$ Value & $\begin{array}{l}\text { Adjusted HR (95\% } \\
\text { CI) }\end{array}$ & $p$ Value $^{\mathrm{a}}$ \\
\hline \multicolumn{7}{|l|}{ All patients } \\
\hline \multicolumn{7}{|l|}{ Esophagectomy } \\
\hline Open & $3691(32.3)$ & $45.0(43.0-46.9)$ & Reference & & & \\
\hline Laparoscopic & 2104 (43.6) & $54.4(50.3-60.4)$ & $0.96(0.91-1.01)$ & 0.1 & Reference & \\
\hline Robotic & $733(44.2)$ & 56.7 (50.9-63.0) & $0.92(0.84-1.00)$ & 0.049 & $0.95(0.87-1.05)$ & 0.3 \\
\hline \multicolumn{7}{|l|}{ Gastrectomy } \\
\hline Open & 8624 (38.9) & $42.5(40.8-44.1)$ & Reference & & & \\
\hline Laparoscopic & 3034 (47.7) & $63.6(58.5-68.5)$ & $0.89(0.85-0.94)$ & $<0.001$ & Reference & \\
\hline Robotic & $884(51.5)$ & $66.4(58.0-84.9)$ & $0.88(0.81-0.96)$ & 0.006 & $0.99(0.90-1.09)$ & 0.8 \\
\hline \multicolumn{7}{|c|}{$\begin{array}{l}\text { Subset analysis in high-volume } \\
\text { centers }\end{array}$} \\
\hline \multicolumn{7}{|l|}{ Esophagectomy } \\
\hline Open & $1072(41.5)$ & $51.7(48.2-57.1)$ & Reference & & & \\
\hline Laparoscopic & $638(54.3)$ & $57.9(48.2-66.5)$ & $1.15(0.98-1.35)$ & 0.1 & Reference & \\
\hline Robotic & $264(58.8)$ & 73.1 (63.0-NR) & $1.72(1.37-2.15)$ & $<0.001$ & $0.89(0.72-1.09)$ & 0.3 \\
\hline \multicolumn{7}{|l|}{ Gastrectomy } \\
\hline Open & $1021(62.8)$ & $63.9(59.0-70.0)$ & Reference & & & \\
\hline Laparoscopic & $2332(52.4)$ & 86.3 (75.3-NR) & $0.95(0.86-1.04)$ & 0.3 & Reference & \\
\hline Robotic & 1114 (60.2) & NR (70.7-NR) & $0.82(0.73-0.91)$ & 0.003 & $0.95(0.89-1.4)$ & 0.7 \\
\hline
\end{tabular}

$H R$ hazard ratio, $C I$ confidence interval; $N R$ not reached

${ }^{a}$ Indicates post-hoc analyses comparing laparoscopic and robotic surgery

significantly better survival than those treated with open gastrectomy (median, 66.4 vs 63.6 vs 42.5 months; $p<$ 0.001; Fig. 2B). After adjustment for potential confounding factors, a significantly longer overall survival was associated with TO (HR $0.58 ; 95 \%$ CI $0.56-0.60 ; p<$ 0.001 ), LAMIG (HR 0.89; 95\% CI 0.85- 0.94; $p<0.001$ ), and RAMIG (HR 0.88; 95\% CI 0.81-0.096; $p=0.006$ ) (Table 2, Supplementary Table S10). However, long-term survival did not differ significantly between RAMIG and LAMIG (HR 0.89; 95\% CI 0.72-1.09; $p=0.3$; Table 3).

Sensitivity Analysis of TO Sensitivity analyses were performed accounting for a hospital stay of 10 days or longer in the TO. The patients receiving RAMIE had a significantly higher TO than those treated with LAMIE or open surgery $(43 \%$ vs $39 \%$ vs $30 \% ; p<0.001$; Supplementary Table S3). The patients achieving TO had a significantly longer survival than those without TO (median, 87.6 vs 35.0 months; $p<0.001$ ). Adjusted analyses showed that the patients receiving RAMIE had significantly longer survival than those treated with open esophagectomy (HR 0.90; 95\% CI 0.82-0.98; $p=0.019$; Supplementary Table S3).
TO High-Volume (Quintile 5) Centers Subset analyses were performed including high-volume centers $(n=6860)$, of which $27 \%(n=1850)$ were LAMIG and $8 \%(n=554)$ were RAMIG. Baseline demographics between open surgery, LAMIG, and RAMIG are presented in Supplementary Table S11. The individual TOs are presented in Supplementary Table S5. The individual rates of TO parameters were significantly higher with RAMIG than with LAMIG or open gastrectomy for margin-negative resections ( $94 \%$ vs $94 \%$ vs $92 \% ; p=$ $0.002), 15$ or more lymph nodes examined (79\% vs $72 \%$ vs $65 \% ; p<0.001)$, and 30 -day readmissions (6\% vs $6 \%$ vs $9 \% ; p<0.001)$, but did not differ for length of stay or 30-day readmissions (Supplementary Table S5).

The similar rates of margin-negative resections between LAMIG and RAMIG may be explained by the higher rates of advanced tumors (cT3/T4) with RAMIG than with LAMIG (60.6\% vs 53.7\%; Supplementary Table S4). The overall prevalence of TOs observed was $56 \%(n=3804)$ and was significantly higher for the patients receiving RAMIG than for those treated with LAMIG or open surgery $(65 \%$ vs $60 \%$ vs $52 \% ; p<0.001$; Table 3$)$. The adjusted analysis showed a greater proportion of TOs for 
(a) Overall Survival

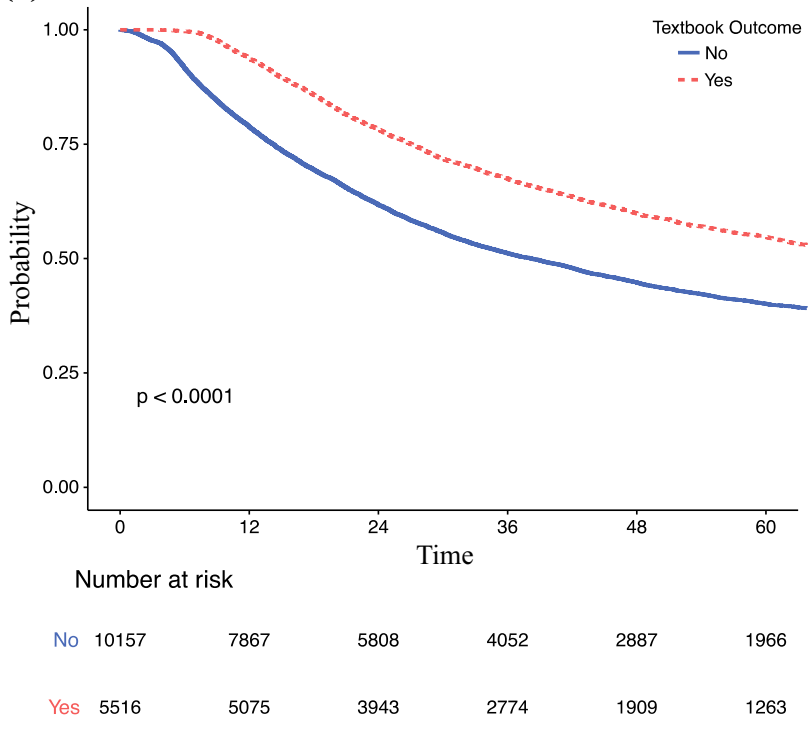

(b) Overall Survival

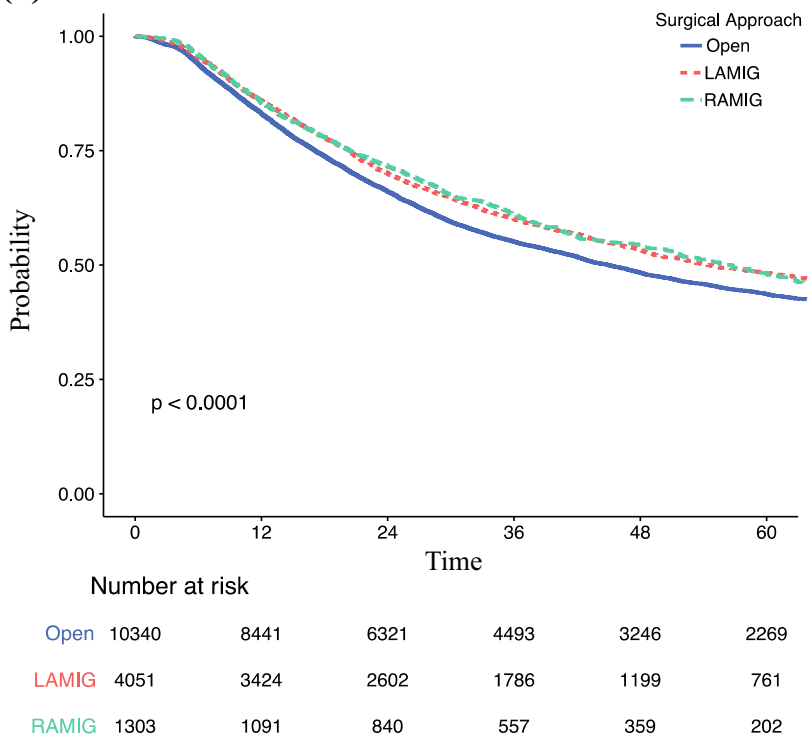

FIG. 1 Overall survival of patients undergoing esophagectomy for esophageal cancer stratified by $\mathbf{A}$ textbook outcome and $\mathbf{B}$ surgical approach.

the patients receiving LAMIG (OR, 1.22; 95\% CI $1.09-1.37 ; p=0.001)$ or RAMIG (OR, $1.45 ; 95 \% \mathrm{CI}$ $1.19-1.76 ; p<0.001)$ than for those treated with open gastrectomy (Supplementary Table S12). The patients achieving TO had a significantly longer survival than those without TO (median, 102.9 vs 45.7 months; $p<0.001$; Fig. 2A). The patients receiving RAMIG or LAMIG had a significantly longer survival than those treated with open esophagectomy (median, NR vs 86.3 vs 63.9 months; $p<$ 0.001; Supplementary Figure S2B). In adjusted analyses, TO (HR: $0.58 ; 95 \%$ CI $0.54-0.63)<0.001$ ) and RAMIG (HR: $0.82 ; 95 \%$ CI 0.73 - 0.91) 0.003) were associated with significantly improved overall survival (Table 3, Supplementary Table S13). However, long-term survival did not differ significantly between RAMIG and LAMIG (HR 0.95 ; 95\% CI 0.89-1.04; $p=0.7$; Table 3).

\section{DISCUSSION}

This national population-based cohort study demonstrated that a robot-assisted minimally invasive approach for esophageal and gastric cancers was associated with an increased rate of TOs and improved long-term survival after adjustment for potential confounding factors, and in subset analysis within high-volume centers. This study suggests that robotic surgery in centers with a sufficient case load may confer some advantages when undertaken by appropriately trained surgeons, and also suggests a rationale for wider dissemination of robotic techniques to improve outcomes from complex esophageal and gastric cancer surgeries. Although the outcomes are comparable between laparoscopic and robotic techniques, strategies to allow dissemination of robotic surgical techniques in the context of complex cancer surgery require careful thought, with credentialing, standardization training programs, audit, and performance evaluation with video analysis before independent practice is permitted. ${ }^{19}$

The rapid increase in adoption of robotic surgery during the past decade is attributable to a few main benefits. First, the robotic technology is thought to improve feasibility and reproducibility, likely shortening the learning curve ${ }^{20}$ compared with the ergonomically challenging laparoscopic approaches. Second, this platform allows for three-dimensional visualization, a magnified view, and improved ergonomics with enhanced stability and maneuverability through the use of articulated wristed instruments controlled from a remote console, with better visualization of tissue planes and deep neurovascular structures. ${ }^{21}$ This allows for a more precise and accurate dissection in narrow spaces, obese patients, and bulky, locally advanced tumors. Difficulties in exposure and the inherent limitations of rigid instrumentation can affect not only the dissection during laparoscopic surgery, but also the completeness of resection margins. Collectively, these advantages translate to lower conversion rates, shorter operative time, fewer postoperative intensive care unit admissions, and a shorter hospital stay. ${ }^{12,22,23}$ Furthermore, robotic surgery appears to be linked with increased odds of margin-negative resection and improved lymphadenectomy, suggesting that these approaches may offer oncologic advantages beyond the benefits of short-term improvements in postoperative recovery. ${ }^{12}$ These marginal gains translate to improvement in long-term survival, as reported in the current study. 
TABLE 4 Baseline clinicopathologic characteristics of patients undergoing gastrectomy for gastric cancer by surgical approach

\begin{tabular}{|c|c|c|c|c|}
\hline & $\begin{array}{l}\text { Open } \\
n(\%)\end{array}$ & $\begin{array}{l}\text { Laparoscopic } \\
n(\%)\end{array}$ & $\begin{array}{l}\text { Robotic } \\
n(\%)\end{array}$ & $p$ Value \\
\hline \multicolumn{5}{|l|}{ Center volume } \\
\hline 1 (lowest) & $3425(15.4)$ & $686(10.8)$ & $143(8.3)$ & \multirow[t]{5}{*}{$<0.001$} \\
\hline 2 & $4525(20.4)$ & 997 (15.6) & $227(13.2)$ & \\
\hline 3 & $4925(22.2)$ & $1135(17.8)$ & $388(22.5)$ & \\
\hline 4 & $4894(22.0)$ & $1702(26.7)$ & $409(23.8)$ & \\
\hline 5 (highest) & $4459(20.1)$ & $1855(29.1)$ & $554(32.2)$ & \\
\hline \multicolumn{5}{|l|}{ Facility type } \\
\hline Community & $8748(39.4)$ & $1897(29.8)$ & 457 (26.6) & \multirow[t]{3}{*}{$<0.001$} \\
\hline Integrated & $3405(15.3)$ & 873 (13.7) & $269(15.6)$ & \\
\hline Academic & $10075(45.3)$ & $3605(56.5)$ & $995(57.8)$ & \\
\hline \multicolumn{5}{|l|}{ Facility location } \\
\hline Northeast & $4853(21.8)$ & 2018 (31.7) & $524(30.4)$ & \multirow[t]{4}{*}{$<0.001$} \\
\hline Midwest & $4868(21.9)$ & $1265(19.8)$ & $327(19.0)$ & \\
\hline South & 8575 (38.6) & $1786(28.0)$ & $552(32.1)$ & \\
\hline West & $3932(17.7)$ & $1306(20.5)$ & $318(18.5)$ & \\
\hline \multicolumn{5}{|c|}{ Hospital distance (miles) } \\
\hline$<12.5$ & $12978(58.4)$ & 3517 (55.2) & $904(52.5)$ & \multirow[t]{3}{*}{$<0.001$} \\
\hline $12.5-49.9$ & 6087 (27.4) & $1829(28.7)$ & $542(31.5)$ & \\
\hline$\geq 50$ miles & $3163(14.2)$ & $1029(16.1)$ & $275(16.0)$ & \\
\hline \multicolumn{5}{|l|}{ Year of diagnosis } \\
\hline 2010-2011 & $6507(29.3)$ & $1163(18.2)$ & $153(8.9)$ & \multirow[t]{4}{*}{$<0.001$} \\
\hline 2012-2013 & $6067(27.3)$ & $1497(23.5)$ & $325(18.9)$ & \\
\hline 2014-2015 & $2738(12.3)$ & 869 (13.6) & $246(14.3)$ & \\
\hline 2016-2017 & $6916(31.1)$ & 2846 (44.6) & 997 (57.9) & \\
\hline \multicolumn{5}{|c|}{ Age at diagnosis (years) } \\
\hline $18-35$ & $240(1.1)$ & $75(1.2)$ & $19(1.1)$ & \multirow[t]{5}{*}{$<0.001$} \\
\hline $36-50$ & $1856(8.4)$ & $486(7.6)$ & $163(9.5)$ & \\
\hline $51-65$ & $7319(33.0)$ & $2185(34.3)$ & $617(35.9)$ & \\
\hline $66-80$ & $9769(44.0)$ & $2846(44.7)$ & $773(45.0)$ & \\
\hline $80+$ & 2997 (13.5) & $769(12.1)$ & $145(8.4)$ & \\
\hline \multicolumn{5}{|l|}{$\operatorname{Sex}$} \\
\hline Male & $15020(67.6)$ & 4478 (70.2) & $1258(73.1)$ & \multirow[t]{2}{*}{$<0.001$} \\
\hline Female & $7208(32.4)$ & $1897(29.8)$ & $463(26.9)$ & \\
\hline \multicolumn{5}{|l|}{ Race } \\
\hline White & $16044(72.2)$ & $4801(75.3)$ & $1311(76.2)$ & \multirow[t]{2}{*}{$<0.001$} \\
\hline Other & $6184(27.8)$ & $1574(24.7)$ & $410(23.8)$ & \\
\hline \multicolumn{5}{|l|}{ CDCC score } \\
\hline 0 & $14291(64.3)$ & $4161(65.3)$ & $1143(66.4)$ & \multirow[t]{4}{*}{0.319} \\
\hline $1-2$ & $7115(32.0)$ & $1977(31.0)$ & $513(29.8)$ & \\
\hline 2 & $1680(7.6)$ & $477(7.5)$ & $109(6.3)$ & \\
\hline $3+$ & $822(3.7)$ & $237(3.7)$ & $65(3.8)$ & \\
\hline \multicolumn{5}{|l|}{ Insurance status } \\
\hline Medicare & $12042(54.8)$ & 3368 (53.5) & $920(53.9)$ & \multirow[t]{4}{*}{$<0.001$} \\
\hline Medicaid & $1685(7.7)$ & $501(8.0)$ & $109(6.4)$ & \\
\hline Private & $7151(32.6)$ & $2232(35.5)$ & $637(37.3)$ & \\
\hline Not insured/other & $1077(4.9)$ & $190(3.0)$ & $40(2.3)$ & \\
\hline \multicolumn{5}{|c|}{ Education level (\%) } \\
\hline$>21$ & $6634(29.8)$ & $1853(29.1)$ & $504(29.3)$ & \multirow[t]{2}{*}{$<0.001$} \\
\hline $13 \%-20.9$ & $5379(24.2)$ & $1392(21.8)$ & $372(21.6)$ & \\
\hline
\end{tabular}


TABLE 4 (continued)

\begin{tabular}{|c|c|c|c|c|}
\hline & $\begin{array}{l}\text { Open } \\
n(\%)\end{array}$ & $\begin{array}{l}\text { Laparoscopic } \\
n(\%)\end{array}$ & $\begin{array}{l}\text { Robotic } \\
n(\%)\end{array}$ & $p$ Value \\
\hline $7 \%-12.9$ & $6089(27.4)$ & $1852(29.1)$ & $516(30.0)$ & \\
\hline$<7$ & $4126(18.6)$ & $1278(20.0)$ & $329(19.1)$ & \\
\hline \multicolumn{5}{|c|}{ Medical income (\$) } \\
\hline 47,999 & $8678(39.0)$ & $2189(34.3)$ & $533(31.0)$ & \multirow[t]{3}{*}{$<0.001$} \\
\hline $48,000-62,999$ & $5298(23.8)$ & 1569 (24.6) & $421(24.5)$ & \\
\hline $63,000+$ & $8252(37.1)$ & $2617(41.1)$ & 767 (44.6) & \\
\hline \multicolumn{5}{|l|}{ Residence } \\
\hline Metro & $18612(83.7)$ & $5331(83.6)$ & $1466(85.2)$ & \multirow[t]{3}{*}{0.493} \\
\hline Urban & $2530(11.4)$ & 738 (11.6) & $173(10.1)$ & \\
\hline Rural & $1086(4.9)$ & $306(4.8)$ & $82(4.8)$ & \\
\hline \multicolumn{5}{|c|}{ AJCC clinical T stage } \\
\hline $\mathrm{cT} 1$ & $3668(16.5)$ & 1385 (21.7) & $328(19.1)$ & \multirow[t]{5}{*}{$<0.001$} \\
\hline cT2 & $2756(12.4)$ & 929 (14.6) & $323(18.8)$ & \\
\hline cT3 & $6525(29.4)$ & 2097 (32.9) & $613(35.6)$ & \\
\hline cT4 & $1444(6.5)$ & $246(3.9)$ & $76(4.4)$ & \\
\hline $\mathrm{cTx}$ & $7835(35.2)$ & $1718(26.9)$ & $381(22.1)$ & \\
\hline \multicolumn{5}{|c|}{ AJCC clinical N stage } \\
\hline cN0 & $11976(53.9)$ & $3687(57.8)$ & $956(55.5)$ & \multirow[t]{5}{*}{$<0.001$} \\
\hline $\mathrm{cN} 1$ & $4433(19.9)$ & $1384(21.7)$ & $461(26.8)$ & \\
\hline $\mathrm{cN} 2$ & $1608(7.2)$ & $445(7.0)$ & $134(7.8)$ & \\
\hline $\mathrm{cN} 3$ & $668(3.0)$ & $118(1.9)$ & $19(1.1)$ & \\
\hline $\mathrm{cNx}$ & $3543(15.9)$ & 741 (11.6) & $151(8.8)$ & \\
\hline \multicolumn{5}{|c|}{ Neoadjuvant therapy } \\
\hline None & $14450(65.0)$ & 3774 (59.2) & $794(46.1)$ & \multirow[t]{3}{*}{$<0.001$} \\
\hline NCRT & $3663(16.5)$ & 1449 (22.7) & $555(32.2)$ & \\
\hline NAC & 4115 (18.5) & $1152(18.1)$ & $372(21.6)$ & \\
\hline \multicolumn{5}{|l|}{ Tumor grade } \\
\hline Well & $1495(6.7)$ & $517(8.1)$ & $125(7.3)$ & \multirow[t]{4}{*}{$<0.001$} \\
\hline Moderate & $7436(33.5)$ & $2372(37.2)$ & $616(35.8)$ & \\
\hline Poor & $11579(52.1)$ & $2950(46.3)$ & $801(46.5)$ & \\
\hline Anaplastic & $1718(7.7)$ & $536(8.4)$ & $179(10.4)$ & \\
\hline \multicolumn{5}{|c|}{ AJCC pathologic T stage } \\
\hline pT0 & $1085(4.9)$ & $453(7.1)$ & $186(10.8)$ & \multirow[t]{6}{*}{$<0.001$} \\
\hline pT1 & $4948(22.3)$ & 1967 (30.9) & $520(30.2)$ & \\
\hline pT2 & $3100(13.9)$ & $973(15.3)$ & 249 (14.5) & \\
\hline pT3 & $7604(34.2)$ & $2011(31.5)$ & $516(30.0)$ & \\
\hline pT4 & $4489(20.2)$ & $722(11.3)$ & $175(10.2)$ & \\
\hline pTx & $1002(4.5)$ & $249(3.9)$ & $75(4.4)$ & \\
\hline \multicolumn{5}{|c|}{ AJCC pathologic $N$ stage } \\
\hline pNO & $9994(48.8)$ & $3474(57.2)$ & $1001(60.6)$ & \multirow[t]{5}{*}{$<0.001$} \\
\hline pN1 & 3984 (19.5) & $1093(18.0)$ & $303(18.3)$ & \\
\hline $\mathrm{pN} 2$ & $3373(16.5)$ & 797 (13.1) & $194(11.7)$ & \\
\hline $\mathrm{pN} 3$ & $1864(9.1)$ & $347(5.7)$ & $76(4.6)$ & \\
\hline $\mathrm{pNx}$ & $1262(6.2)$ & $367(6.0)$ & $78(4.7)$ & \\
\hline \multicolumn{5}{|c|}{ Lymphovascular invasion } \\
\hline Absent & 10435 (46.9) & 3467 (54.4) & $963(56.0)$ & \multirow[t]{2}{*}{$<0.001$} \\
\hline Present & 8377 (37.7) & 1945 (30.5) & 458 (26.6) & \\
\hline
\end{tabular}


TABLE 4 (continued)

\begin{tabular}{lccc}
\hline & $\begin{array}{l}\text { Open } \\
n(\%)\end{array}$ & $\begin{array}{l}\text { Laparoscopic } \\
n(\%)\end{array}$ & $\begin{array}{l}\text { Robotic } \\
n(\%)\end{array}$ \\
\hline $\begin{array}{l}\text { Unknown } \\
\text { 30-Day mortality }\end{array}$ & $3416(15.4)$ & $963(15.1)$ & $300(17.4)$ \\
No & $21259(95.6)$ & $6203(97.3)$ & $1683(97.8)$ \\
Yes & $969(4.4)$ & $172(2.7)$ & $38(2.2)$ \\
\hline
\end{tabular}

CDCC Charlson-Deyo Score, AJCC American Joint Committee on Cancer, NCRT neoadjuvant chemoradiotherapy, NAC neoadjuvant chemotherapy

(a) Overall Survival

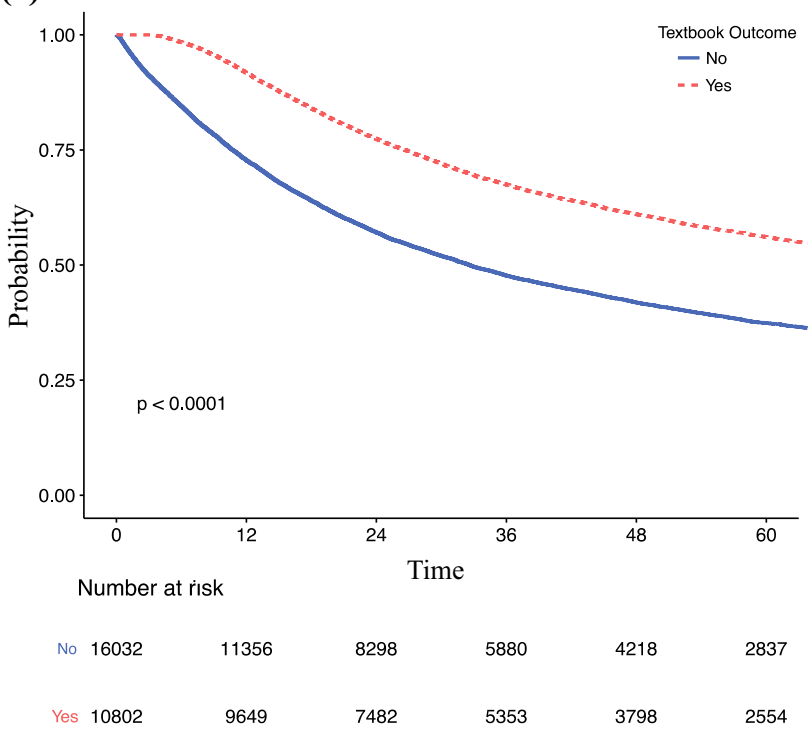

(b) Overall Survival

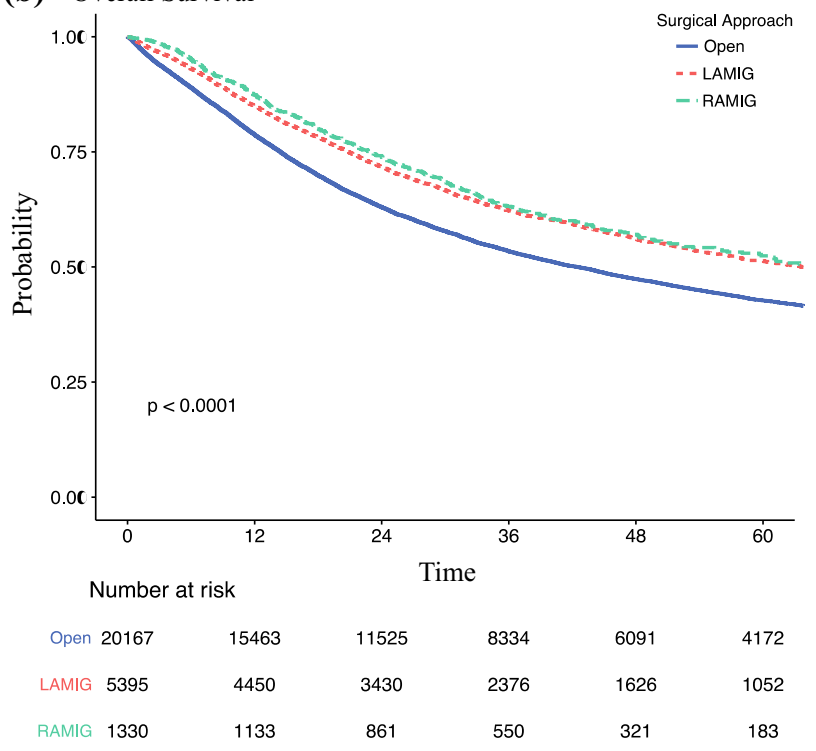

FIG. 2 Overall survival of patients undergoing gastrectomy for gastric cancer stratified by $\mathbf{A}$ textbook outcome and B surgical approach.
During the next few years, dissemination of robotic surgery is key to ensuring its routine adoption into clinical practice to optimize patient benefits. First, implementation of training programs should be safe and be adopted in highvolume centers and/or surgeons to ensure a critical case load to shorten any potential proficiency gain curve. Second, regulators and the surgical community need to have systems for tightly controlled auditing, such as an international registry, in place to monitor performance of robotic surgeries across various specialties. Because accurate data are necessary to inform the creation of appropriate safeguards, national bodies should consider providing coverage for robotic surgery with provisions for evidence development. ${ }^{24}$ The Upper GI International Robotic Association (UGIRA) was established to facilitate the reporting of robotic procedures worldwide and to analyze variation and learning curves. ${ }^{3,5,19}$ Use of these provisions would facilitate greater understanding of how robotic procedures are used in real-world practice. Akin to post-market surveillance of pharmaceuticals, such provisions also would create a common data resource from which the comparative safety and effectiveness of robotic operations could be evaluated by numerous investigators. Third, video-based analyses of performance and telemedicine for surgical coaching should be used to shorten the learning curve among surgeons. Finally, additional adjuncts such as image-based surgery with projections of preoperative imaging may allow refined surgical anatomy and dissection in cancer surgery.

This study had some important limitations. First, certain inherent biases with access to hospital services offering robotic surgery may be associated with TOs such as improved oncology services, access to research trials, and improved failure to rescue. These biases may contribute to overall better outcomes. However, these data provide us impetus for future qualitative research that may help explain such differences. Second, the NCDB does not distinguish whether longitudinal (proximal and distal) or circumferential margins were involved, prohibiting ability 
to assess their relative importance. Third, the observed findings may relate to a cluster effect whereby patients likely to receive a robotic procedure are likely to have better performance status and be more likely to travel to academic centers. ${ }^{25}$ Fourth, understanding the health economic costs of the robotic platform is key. Although robotic surgery in this study was associated with higher rates of TOs through reduced overall complications and need for intervention or return to the intensive treatment unit than open or laparoscopic surgery, robotic surgery likely may have lower costs to the hospitals in the long term. However, these data are not available in the NCDB, which mandates further research. Finally, patient-related outcomes such as quality of life were not included in the TOs, which could be important because desired outcomes might differ between physicians and patients. ${ }^{26}$

\section{CONCLUSION}

Despite potentially adverse learning curve effects and more advanced tumor stages captured during the study period, both RAMIE and RAMIG, as performed in mostly high-volume centers, were associated with improved TOs and long-term survival. Therefore, consideration for their wider adoption but also a well-designed phase 3 RCT is required for a full evaluation of the benefits conferred by robotic techniques for esophageal and gastric cancers.

DISCLOSURE The authors declare that they have no conflict of interest.

Supplementary Information The online version contains supplementary material available at https://doi.org/10.1245/s10434021-11082-y.

OPEN ACCESS This article is licensed under a Creative Commons Attribution 4.0 International License, which permits use, sharing, adaptation, distribution and reproduction in any medium or format, as long as you give appropriate credit to the original author(s) and the source, provide a link to the Creative Commons licence, and indicate if changes were made. The images or other third party material in this article are included in the article's Creative Commons licence, unless indicated otherwise in a credit line to the material. If material is not included in the article's Creative Commons licence and your intended use is not permitted by statutory regulation or exceeds the permitted use, you will need to obtain permission directly from the copyright holder. To view a copy of this licence, visit http://creativecommons. org/licenses/by/4.0/.

\section{REFERENCES}

1. Kolfschoten NE, Kievit J, Gooiker GA, van Leersum NJ, Snijders HS, Eddes EH, et al. Focusing on desired outcomes of care after colon cancer resections; hospital variations in "textbook outcome.” Eur J Surg Oncol. 2013;39(2):156-63.

2. Mehta R, Paredes AZ, Tsilimigras DI, Moro A, Sahara K, Farooq $A$, et al. Influence of hospital teaching status on the chance to achieve a textbook outcome after hepatopancreatic surgery for cancer among Medicare beneficiaries. Surgery. 2020;168:92-100.

3. Van Boxel GI, Kingma BF, Ruurda JP. Formal assessment of the learning curve for minimally invasive methods is vital in retrospective cohort studies. Am J Obstet Gynecol. 2020;222:95-6.

4. van der Sluis PC, van der Horst S, May AM, Schippers C, et al. Robot-assisted minimally invasive thoracolaparoscopic esophagectomy versus open transthoracic esophagectomy for resectable esophageal cancer: a randomized controlled trial. Ann Surg. 2019;269:621-30.

5. Kingma BF, Grimminger PP, van der Sluis PC, van Det MJ, Kouwenhoven EA, Chao YK, et al. US worldwide techniques, outcomes in robot-assisted minimally invasive esophagectomy (RAMIE): results from the Multicenter International Registry. Ann Surg. 2020. https://doi.org/10.1097/SLA. 0000000000004550.

6. Bobo Z, Xin W, Jiang L, Quan W, Liang B, Xiangbing D, Ziqiang W. Robotic gastrectomy versus laparoscopic gastrectomy for gastric cancer: meta-analysis and trial sequential analysis of prospective observational studies. Surg Endosc. 2019;33:1033-48.

7. van Boxel GI, Ruurda JP, van Hillegersberg R. Robotic-assisted gastrectomy for gastric cancer: a European perspective. Gastric Cancer. 2019;22:909-19.

8. Merath K, Chen Q, Bagante F, Beal E, Akgul O, Dillhoff M, et al. Textbook outcomes among medicare patients undergoing hepatopancreatic surgery. Ann Surg. 2020;271:1116-23.

9. Salet N, Bremmer RH, Verhagen M, Ekkelenkamp VE, Hansen BE, de Jonge PJF, de Man RA. Is Textbook outcome a valuable composite measure for short-term outcomes of gastrointestinal treatments in the Netherlands using hospital information system data? A retrospective cohort study. BMJ Open. 2018;8:e019405.

10. van Roessel S, Mackay TM, van Dieren S, van der Schelling GP, et al. Dutch Pancreatic Cancer G. Textbook outcome: nationwide analysis of a novel quality measure in pancreatic surgery. Ann Surg. 2020;271:155-62.

11. Kalff MC, Vesseur I, Eshuis WJ, Heineman DJ, Daams F, van der Peet DL, et al. The association of textbook outcome and longterm survival after esophagectomy for esophageal cancer. Ann Thorac Surg. 2020;112:1134-41.

12. Kulshrestha S, Bunn C, Patel PM, Sweigert PJ, Eguia E, Pawlik TM, Baker MS. Textbook oncologic outcome is associated with increased overall survival after esophagectomy. Surgery. 2020;168:953-61.

13. Levy J, Gupta V, Amirazodi E, Allen-Ayodabo C, Jivraj N, Jeong Y, et al. Textbook outcome and survival in patients with gastric cancer: an analysis of the Population Registry of Esophageal and Stomach Tumours in Ontario (PRESTO). Ann Surg. 2020. http s://doi.org/10.1097/SLA.0000000000003849.

14. Bilimoria KY, Bentrem DJ, Ko CY, Ritchey J, Stewart AK, Winchester DP, Talamonti MS. Validation of the 6th-edition AJCC pancreatic cancer staging system: report from the National Cancer Database. Cancer. 2007;110:738-44.

15. Merkow RP, Rademaker AW, Bilimoria KY. Practical guide to surgical data sets: National Cancer Database (NCDB). JAMA Surg. 2018;153:850-1.

16. Quan H, Li B, Couris CM, Fushimi K, Graham P, Hider P, et al. Updating and validating the Charlson Comorbidity Index and score for risk adjustment in hospital discharge abstracts using data from 6 countries. Am J Epidemiol. 2011;173:676-82.

17. Busweiler LA, Schouwenburg MG, van Berge Henegouwen MI, Kolfschoten NE, de Jong PC, Rozema T, et al. Dutch Upper Gastrointestinal Cancer Audit, g: textbook outcome as a composite measure in oesophagogastric cancer surgery. Br J Surg. 2017;104:742-50. 
18. Kamarajah SK, Sonnenday CJ, Cho CS, Frankel TL, Bednar F, Lawrence TS, Nathan H. Association of adjuvant radiotherapy with survival after margin-negative resection of pancreatic ductal adenocarcinoma: a propensity-matched National Cancer Database (NCDB) analysis. Ann Surg. 2019;22(1):10-22.

19. Kingma BF, Hadzijusufovic E, Van der Sluis PC, Bano E, Lang $\mathrm{H}$, Ruurda JP, et al. A structured training pathway to implement robot-assisted minimally invasive esophagectomy: the learning curve results from a high-volume center. Dis Esophagus. 2020;33(Suppl 2):doaa047.

20. van der Sluis PC, Ruurda JP, van der Horst S, Goense L, van Hillegersberg R. Learning curve for robot-assisted minimally invasive thoracoscopic esophagectomy: results from 312 cases. Ann Thorac Surg. 2018;106:264-71.

21. Wexner SD, Bergamaschi R, Lacy A, Udo J, Brolmann H, Kennedy RH, John $\mathrm{H}$. The current status of robotic pelvic surgery: results of a multinational interdisciplinary consensus conference. Surg Endosc. 2009;23:438-43.

22. Luketich JD, Alvelo-Rivera M, Buenaventura PO, Christie NA, McCaughan JS, Litle VR, et al. Minimally invasive esophagectomy: outcomes in 222 patients. Ann Surg. 2003;238:486-94.
23. Nguyen NT, Follette DM, Wolfe BM, Schneider PD, Roberts P, Goodnight JE Jr. Comparison of minimally invasive esophagectomy with transthoracic and transhiatal esophagectomy. Arch Surg. 2000;135:920-5.

24. Daniel GW, Rubens EK, McClellan M. Coverage with evidence development for Medicare beneficiaries: challenges and next steps. JAMA Intern Med. 2013;173:1281-2.

25. Speicher PJ, Englum BR, Ganapathi AM, Wang X, Hartwig MG, D'Amico TA, Berry MF. Traveling to a high-volume center is associated with improved survival for patients with esophageal cancer. Ann Surg. 2017;265:743-9.

26. de Bekker-Grob EW, Niers EJ, van Lanschot JJ, Steyerberg EW, Wijnhoven BP. Patients' preferences for surgical management of esophageal cancer: a discrete choice experiment. World J Surg. 2015;39:2492-9.

Publisher's Note Springer Nature remains neutral with regard to jurisdictional claims in published maps and institutional affiliations. 